\title{
Lifetimes of the $K_{S}$ and $K_{L}$ mesons and the absolute $K^{+} \rightarrow \pi^{+} \pi^{-} \pi^{+}$branching ratio with KLOE.
}

\author{
Simona Serena Bocchetta* on behalf of KLOE collaboration ${ }^{\dagger}$ \\ Dipartimento di Fisica e INFN Sezione di "Roma Tre", Rome, Italy \\ E-mail: bocchettadroma3.infn.it
}

\begin{abstract}
The KLOE experiment at the Frascati $e^{+} e^{-}$collider DAФNE, operating at the $\phi$ peak, provides unique opportunities for precision measurements of kaon properties. We present the $K_{S}$ lifetime measurement, performed with $\sim 20$ million events and discuss the obtainable accuracy. A preliminary update of the $K_{L}$ lifetime measurement is also presented, $\tau_{K_{L}}=50.56 \pm 0.14_{\text {stat }} \pm 0.21_{\text {syst }}$ ns, from a sample of $\sim 46$ million $K_{L} \rightarrow \pi^{0} \pi^{0} \pi^{0}$ events, tagged by $K_{S} \rightarrow \pi^{+} \pi^{-}$decays. The present error on the $K_{L}$ lifetime still limits the accuracy of the determination of the $V_{u s}$ CKM matrix element. We also discuss studies of the branching ratio for $K^{+} \rightarrow \pi^{+} \pi^{-} \pi^{+}$using approximately $10 \%$ of all KLOE data. This measurement would supersede the previous old result dating back to 1972 and would complete the KLOE program of precise and fully inclusive measurements of the $K^{ \pm}$ dominant branching ratios.
\end{abstract}

2009 KAON International Conference KAON09,

June 09 - 122009

Tsukuba, Japan

* Speaker.

${ }^{\dagger}$ F. Ambrosino, A. Antonelli, M. Antonelli, F. Archilli, P. Beltrame, G. Bencivenni, C. Bini, C. Bloise, S. Bocchetta, F. Bossi, P. Branchini, G. Capon, D. Capriotti, T. Capussela, F. Ceradini, P. Ciambrone, E. De Lucia, A. De Santis, P. De Simone, G. De Zorzi, A. Denig, A. Di Domenico, C. Di Donato, B. Di Micco, M. Dreucci, G. Felici, S. Fiore, P. Franzini, C. Gatti, P. Gauzzi, S. Giovannella, E. Graziani, M. Jacewicz, V. Kulikov, G. Lanfranchi, J. Lee-Franzini, M. Martini, P. Massarotti, S. Meola, S. Miscetti, M. Moulson, S. Müller, F. Murtas, M. Napolitano, F. Nguyen, M. Palutan, A. Passeri, V. Patera, P. Santangelo, B. Sciascia, A. Sibidanov, T. Spadaro, L. Tortora, P. Valente, G. Venanzoni, R. Versaci. 


\section{The KLOE experiment}

The KLOE detector operates at DAФNE, the Frascati $e^{+} e^{-}$collider working at a center of mass energy $W \sim m_{\phi} \sim 1.02 \mathrm{GeV}$. $\phi$ mesons are produced with a visible cross section of $\sim 3.1 \mu \mathrm{b}$ and a transverse momentum of $\sim 12.5 \mathrm{MeV} / \mathrm{c}$ directed toward the center of the collider rings. The energy $W$, the coordinates of the beam's interaction point (IP) and the $\phi$ momentum are determined, run by run, with high precision, from Bhabha scattering events.

The KLOE detector consists of a large drift chamber (DC), surrounded by a electromagnetic calorimeter, EMC. A superconducting coil provides an axial magnetic field of $0.52 \mathrm{~T}$. The DC [1], a cylinder of $4 \mathrm{~m}$ diameter and $3.3 \mathrm{~m}$ in length provides good measurements of $K_{L}$ decays over a length of $\mathcal{O}(1 / 3)$ of their lifetime. The momentum resolution for tracks at large polar angle is $\sigma\left(p_{\perp}\right) / p_{\perp} \sim 0.4 \%$. Secondary vertices are reconstructed with a resolution of $\sim 3 \mathrm{~mm}$. The EMC [2] is a lead-scintillating fiber sampling calorimeter consisting of a barrel and two endcaps, which cover $98 \%$ of the solid angle. Photon energies and arrival times are measured with the EMC with resolutions $\sigma_{E} / E \sim 5.7 \% / \sqrt{E(\mathrm{GeV})}$ and $\sigma_{t}=54 / \sqrt{E(\mathrm{GeV})} \oplus 50 \mathrm{ps}$, respectively.

The trigger [⿰]] uses calorimeter and drift chamber information. The calorimeter trigger requires two local energy deposits above threshold (cosmic-ray rejection is performed at this level). The DC trigger is based on the multiplicity and topology of the hits in the drift cells. The trigger is synchronized with the accelerating radio frequency divided by 4 ( $T=10.8 \mathrm{~ns})$. The bunch crossing period at DA $\Phi N E$ for most of our data is $2.7 \mathrm{~ns}$. The correct collision time $T_{0}$ of the event is determined offline, during event reconstruction [ [ of the total data collected between 2002 and 2005, corresponding to an integrated luminosity of $\sim 2.5 \mathrm{fb}^{-1}$.

$\phi$-mesons decay to $K_{S} K_{L}\left(K^{+} K^{-}\right)$pairs $\sim 34 \%(\sim 49 \%)$ of the time. The $K$ mesons are produced in a pure $J^{P C}=1^{--}$state, so that observation of a $K_{S}\left(K^{+}\right)$in an event signals (tags) the presence of a $K_{L}\left(K^{-}\right)$and vice-versa. Pure, monochromatic, $K_{S}$ and $K_{L}$ beams are therefore available to KLOE. The $K_{S} \rightarrow \pi^{+} \pi^{-}$decay can be selected requiring two tracks of opposite sign and an invariant mass consistent with $M_{K}$. The efficiency, mainly geometrical, is $\sim 70 \%$. The angular resolution on $K_{S}$ direction is $\sim 1^{\circ}$ and the momentum resolution from the measured track momenta $\left(\mathbf{p}_{K_{S}}=\mathbf{p}_{\pi^{+}}+\mathbf{p}_{\pi^{-}}\right)$is $\sim 1 \mathrm{MeV}$. The kaon momentum can also be determined from the $\phi \rightarrow K^{0} \overline{K^{0}}$ decay kinematics. Requiring compatibility between the two measurement improves purity and accuracy. $K_{S}$ and $K_{L}$ are distinguishable from their lifetimes. The average decay paths are $\lambda_{S} \sim 0.6 \mathrm{~cm}$ and $\lambda_{L} \sim 340 \mathrm{~cm}$.

Because of all the above, KLOE is the only experiment that can simultaneously measure the complete set of experimental inputs (branching ratios, lifetimes and form factors parameters), necessary for the calculation of $\left|V_{\mathrm{us}}\right|$ from both charged and neutral kaons, see ref. [5]. In addition, KLOE is the only experiment with a true, pure $K_{S}$-beam and can therefore measure $K_{S}$ branching ratios well below the $10^{-4}$ level, [6].

The average position of the $\phi$ production point $\mathbf{x}_{\phi}$ is obtained run-by-run from Bhabha scattering events, with spatial resolutions of $\sigma_{x y z} \sim 1 \mathrm{~mm}$. The luminous point has spread in $z$ of $\sim 1.5$ $\mathrm{cm}$. A better determination of the $z$ coordinate of the decay point along the beam line is evaluated event-by-event by using the point of closest approach of the $K_{S}$ line of flight propagated backwards from the $K_{S}$ vertex to the beam axis, reaching in this way a resolution of $\sim 0.2 \mathrm{~cm}$, see fig. 1 (left). 

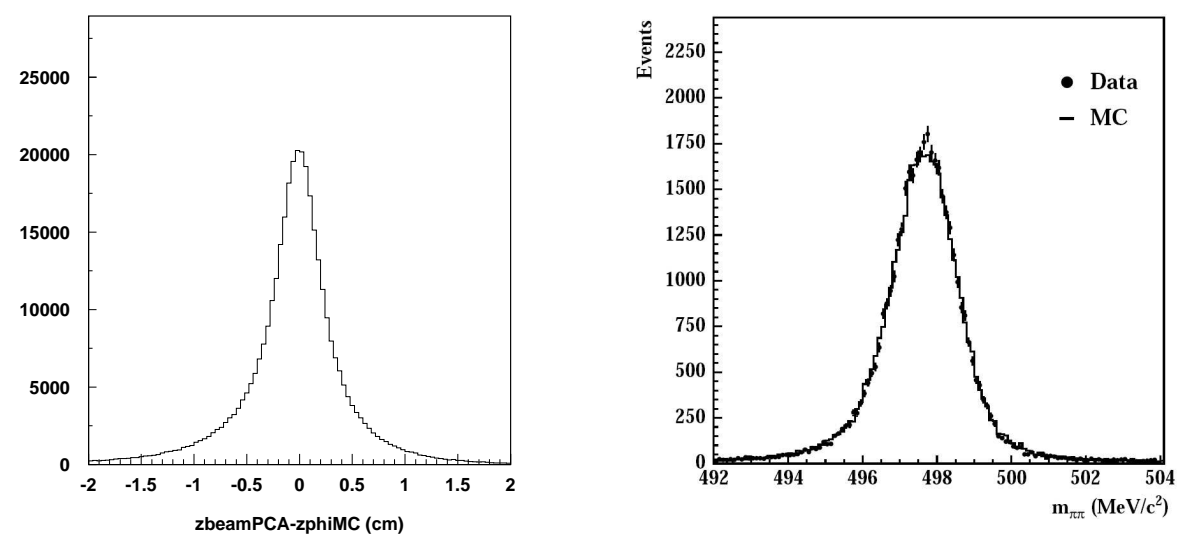

Figure 1: Left: Resolution on $z_{\phi}$ coordinate. Right: Invariant mass of two charged tracks from $K_{S}$ decay.

\section{2. $K_{S}$ lifetime.}

KLOE measures the $K_{S}$ lifetime with a pure $K_{S}$ beam and event-by-event knowledge of the $K_{S}$ momentum. $\tau\left(K_{S}\right)$ can be measured as a function of sidereal time [7] which is interesting for tests of quantum mechanics, $C P T$ and Lorentz invariance.

The lifetime is obtained by fitting the proper time, $t^{*}$, distribution of $K_{S} \rightarrow \pi^{+} \pi^{-}$decays. The $t^{*}$ resolution after event reconstruction, fig. 2(left), is not sufficient for obtaining a lifetime accuracy of $0.1 \%$. Resolution can be much improved by: 1) reconstruction of the IP event-by-event using a geometrical fit; 2) selecting events with pions decaying at large angle with respect to the $K_{S}$ path; 3) rejecting poorly measured tracks by a cut on the the track fit $\chi^{2}$ value. The $t^{*}$ resolution improves as shown in fig. 2, right.
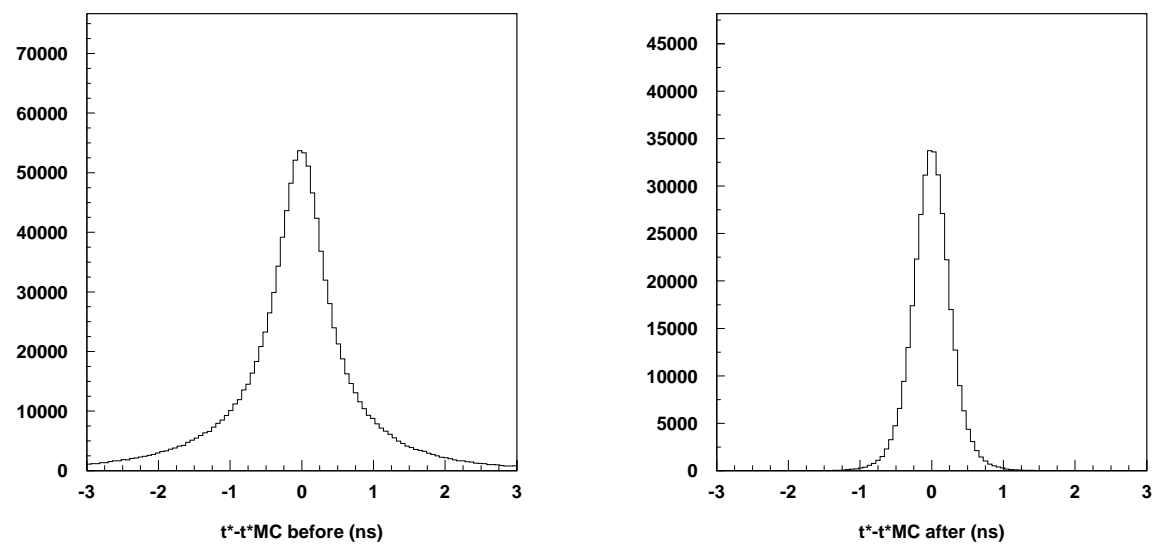

Figure 2: Time resolution before (left) and after (right) event quality selection.

Since the resolution depends on the $K_{S}$ direction, we fit to the proper time distribution for each of 270 bins in $\cos \theta_{K}$ and $\phi_{K}$. The fit range runs from -2 to $7 \times \tau_{S}$, and uses an exponential convoluted with two gaussians (four parameters: lifetime, relative normalizations and two widths), see fig. 3, left. The statistical error on $\tau\left(K_{S}\right)$ is less than $0.1 \%$. The efficiency is $\sim 13 \%$, fig. 3, right. Low efficiency regions, $\left|\cos \theta_{K}\right|>0.7$, are not used. 

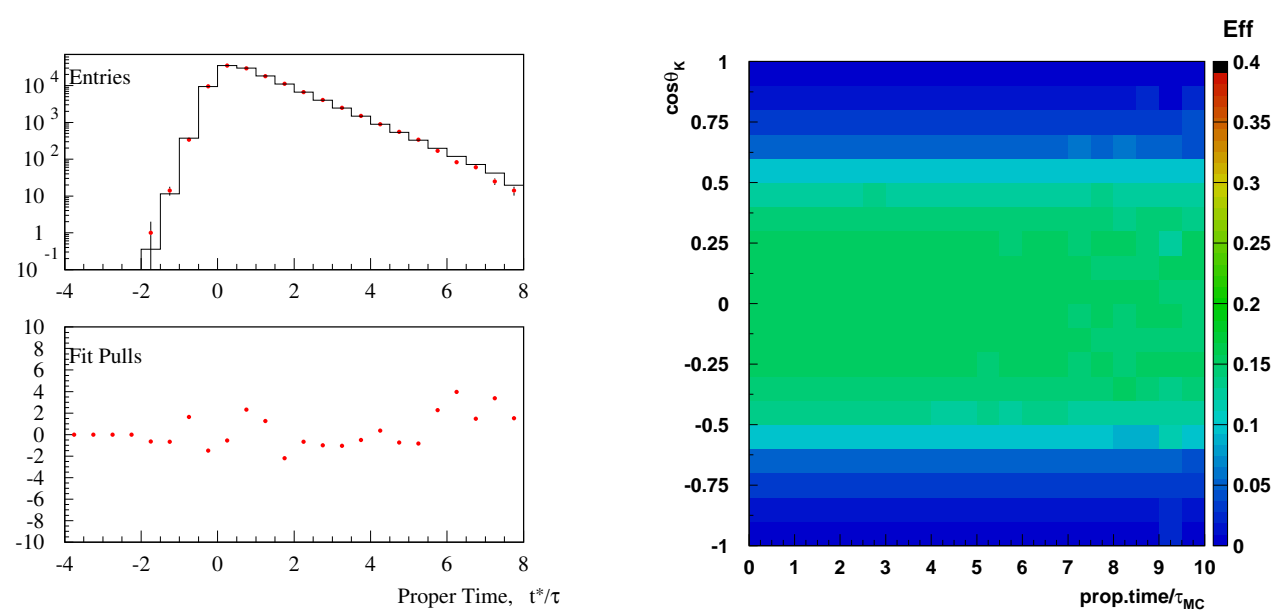

Figure 3: Left: Fit to the $K_{S}$ proper time distribution (top) and fit pulls (bottom). Right: Efficiency as a function of $\cos \theta_{K}$ and proper time.

Table 1 shows a summary of the systematic errors, a total error competitive with the precise measurements from $\mathrm{KTeV}$ [8] [9] and NA48 [10] is expected.

\begin{tabular}{|c|c|}
\hline Source & $\delta \tau_{S} / \tau_{S} \times 10^{-4}$ \\
\hline Selection cuts & 3.3 \\
Acceptance $\cos \theta_{K}$ cut & 5.7 \\
Kaon mass uncertainty [11] & 0.4 \\
Fit range & 5.0 \\
\hline
\end{tabular}

Table 1: Summary of $K_{S}$ lifetime measurement systematic uncertainties.

\section{3. $K_{L}$ lifetime.}

The $K_{L}$ lifetime is fundamental to the determination of the CKM matrix element $\left|V_{\text {us }}\right|$ from $K_{L}$ semileptonic decays and its error remains the limiting factor on the $\left|V_{\mathrm{us}}\right|$ accuracy. Using all the available data, KLOE can improve statistical and systematic error over its previous measurement [12] $\tau\left(K_{L}\right)=50.92 \pm 0.17_{\text {stat }} \pm 0.25_{\text {syst }}$ ns, obtained with $\sim 15$ million $K_{L}$ decays. KLOE also performed a $K_{L}$ decay rate measurement [13] by counting the number of decays in a fiducial volume obtaining $\tau\left(K_{L}\right)=50.72 \pm 0.11_{\text {stat }} \pm 0.35_{\text {syst }}$ ns. We present here a preliminary measurement based on 46 million $K_{L} \rightarrow 3 \pi^{0}$ events.

$K_{L}$ mesons are tagged by detecting $K_{S} \rightarrow \pi^{+} \pi^{-}$decays and the time dependence of the $K_{L} \rightarrow$ $\pi^{0} \pi^{0} \pi^{0}$ decays is used to measure the $K_{L}$ lifetime. The $3 \pi^{0}$ mode is chosen because is the most abundant, has high detection efficiency and the tagging efficiency is almost independent of the $K_{L}$ path length. We require the following for the tagging $K_{S} \rightarrow \pi^{+} \pi^{-}$decays. 1). There must be two opposite curvature tracks originating from a point $C$ inside a cylinder, centered at the IP, of $10 \mathrm{~cm}$ radius and $|z|<20 \mathrm{~cm} .2$ ). The $K_{S}$ momentum in the $\phi$ rest frame satisfies $100<p\left(K_{S}\right)<120$ $\mathrm{MeV} / \mathrm{c}$. 3). The dipion invariant mass lies in the interval $482.6-512.6 \mathrm{MeV} / \mathrm{c}^{2}$. The efficiency for finding $K_{S} \rightarrow \pi^{+} \pi^{-}$decays is $\sim 68 \%$. 

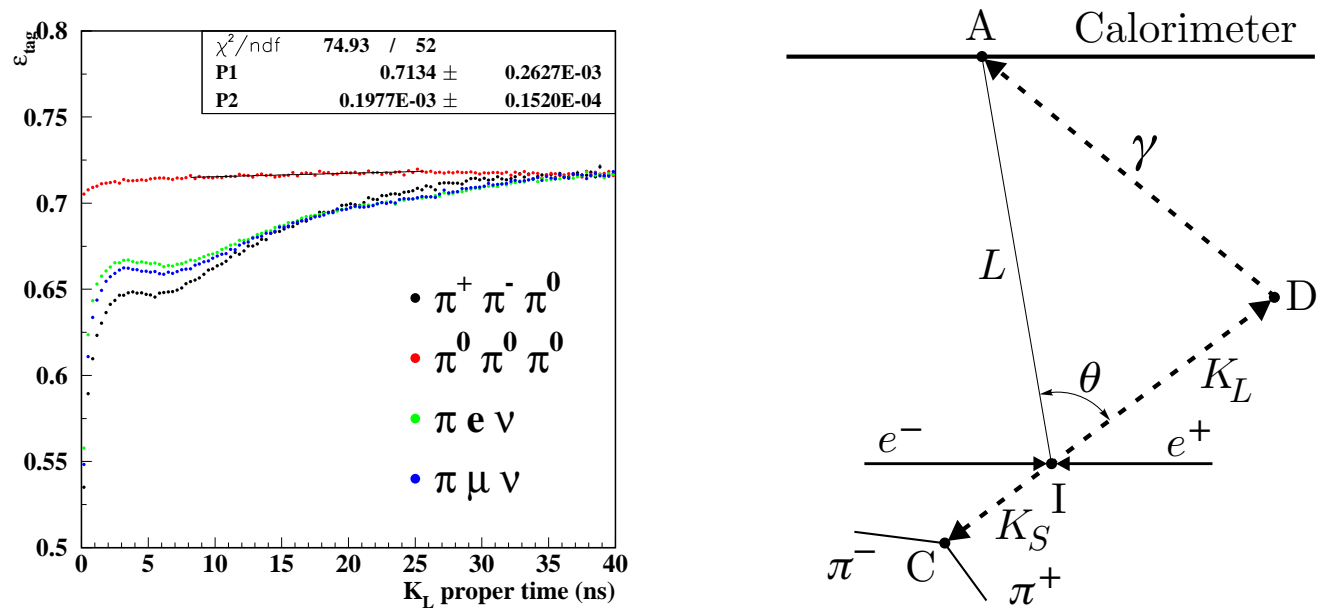

Figure 4: Left: Tagging efficiency. Right: Single photon geometry.

The position of the $K_{L} \rightarrow 3 \pi^{0}$ decay point is obtained from the photon arrival times at the EMC. Each photon defines a triangle IDA, see fig. \#, right. I is the interaction point, D the $K_{L}$ decay point and $\mathrm{A}$ the photon entry point in the calorimeter. From the known positions of I and A, the $\widehat{\mathrm{AID}}=\theta$ angle and the time spent by the kaon and the photon to cover the path IDA we find the length of ID. There are two solutions. One of them corresponds to D being along the $K_{S}$ path and is rejected. The path length of the $K_{L}$-meson is obtained from the energy weighted average of all $l_{K, i}$, $\left\langle l_{K}\right\rangle=\sum\left(l_{K, i} E_{i}\right) / \sum E_{i}$ where $i$ is the photon index. Finally we require at least three photons with $\left|l_{K, i}-l_{K}\right|<5 \sigma\left(l_{K}\right)$.

The single photon vertex resolution $\sigma_{l_{K}}$ is measured from data as a function of the photon energy. Fig. 5 (left) shows the comparison between the photon multiplicity obtained with this method for data (black squares) and Monte Carlo (red dots). After the multi-photon neutral vertex recon-
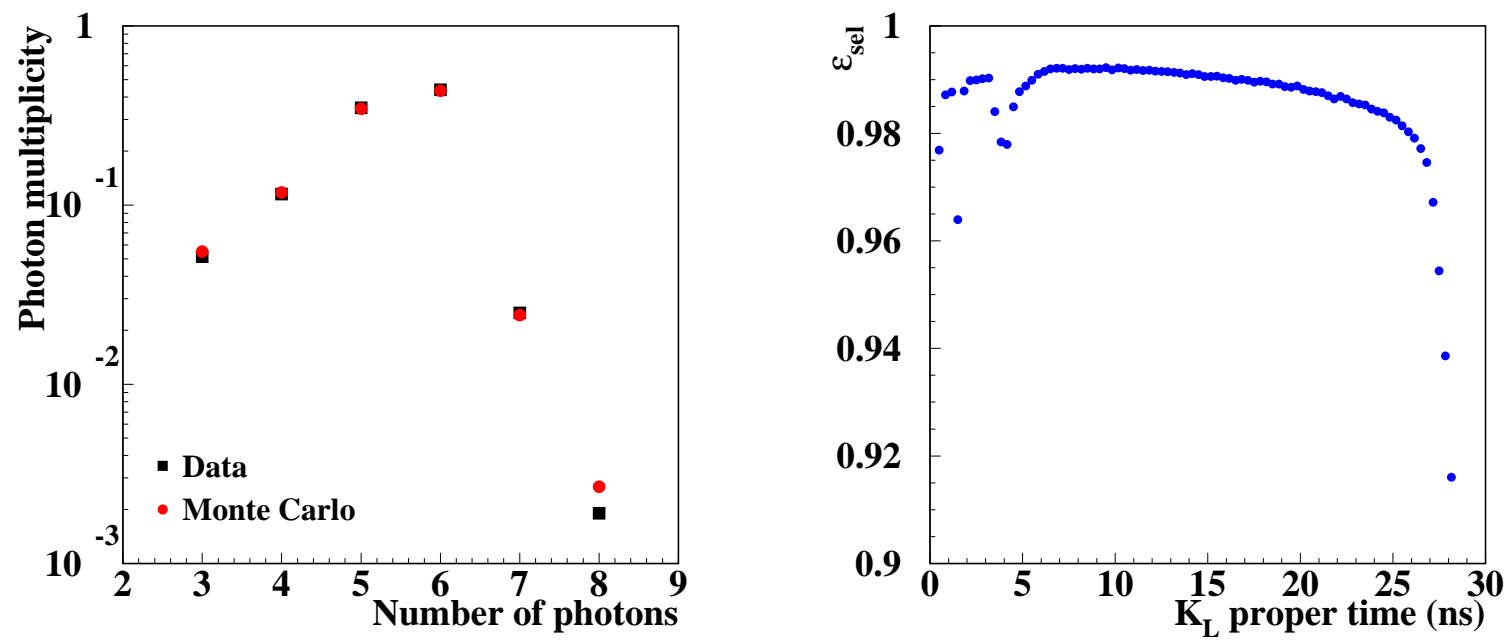

Figure 5: Left: Photon multiplicity for data (black squares) and Monte Carlo (red dots). Right: Signal selection efficiency after all cuts. 
struction, the residual background is rejected using ad-hoc topological variables; the background contamination after these cuts is $\sim 1.81 \%$, essentially given by $K_{L} \rightarrow \pi^{+} \pi^{-} \pi^{0}$ decays, regeneration and nuclear interactions in events with $\mathrm{N}=3,4$ photons, while for $\mathrm{N} \geq 5$ the sample of $K_{L} \rightarrow \pi^{0} \pi^{0} \pi^{0}$ decays is almost pure. The total acceptance is evaluated by Monte Carlo and corrected with data, is very close to 1 and almost constant over a wide decay region, as shown in fig. 5 (right).

The decay $K_{L} \rightarrow \pi^{+} \pi^{-} \pi^{0}$, selected by means of tight kinematic cuts, has been used as a control sample to study the neutral vertex reconstruction quality:

1. the accuracy of the $l_{K}$ determination from a single photon was checked by comparing it with the decay vertex obtained by DC tracking information. This allowed also to perform a fine calibration of the calorimeter time scale;

2. the single $\gamma$ decay vertex spatial resolution has also been measured in the control sample as a function of the $K_{L}$ path and of the photon energy, the average value is $\sigma_{l_{K}} \sim 2.2 \mathrm{~cm}$;

3. the single $\gamma$ reconstruction efficiency has been measured, $\epsilon_{\gamma}=N_{\gamma \text {, rec }} / N_{\gamma}$, tag where $N_{\gamma \text {, tag }}$ is the number of $K_{L} \rightarrow \pi^{+} \pi^{-} \pi^{0}$ events in which at least one photon is detected, and $N_{\gamma}$, rec is the number of events in which a second photon is detected where we expect to find it from kinematics. The fig. 0 shows the efficiency as a function of the transverse radius $R_{t}$ of the $K_{L}$ neutral vertex (left) and as a function of the expected photon energy $E_{\gamma}$ (right). The Monte Carlo has been corrected with the efficiencies ratio $\epsilon_{d t} / \epsilon_{M C}$ using a hit-or-miss method taking into account the dependencies on $R_{t}$ and $E_{\gamma}$.
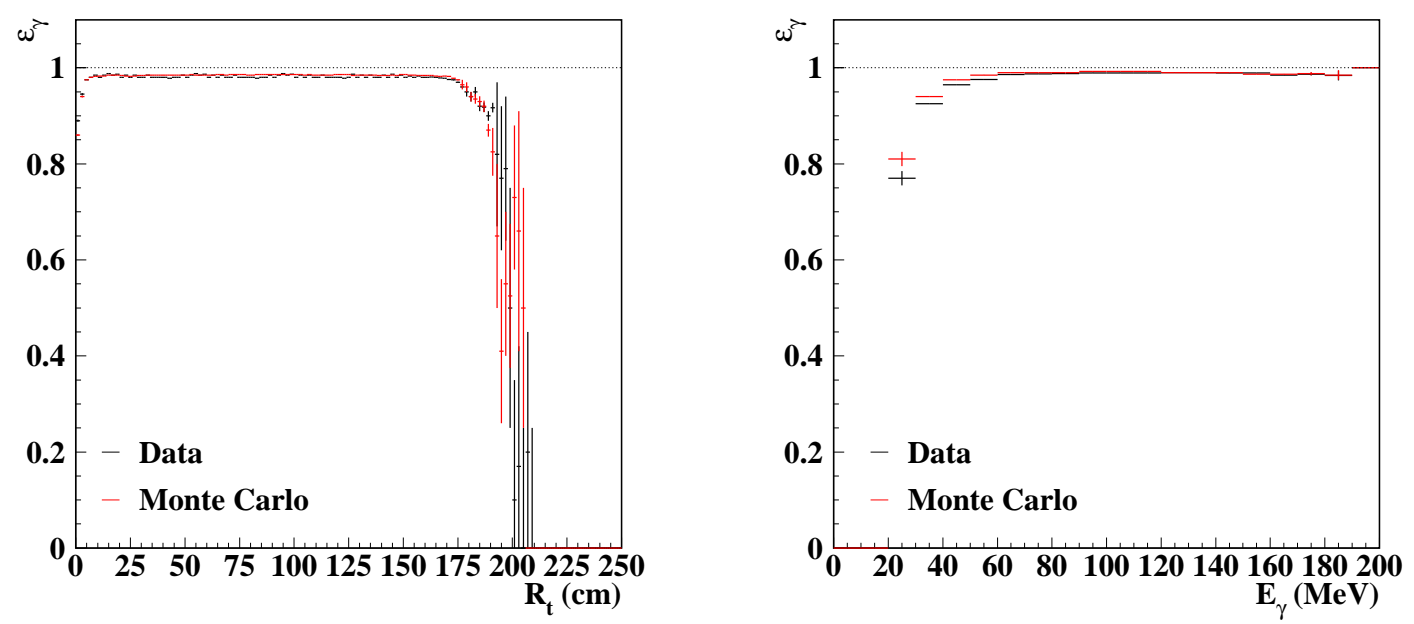

Figure 6: Single-photon vertex reconstruction efficiency as a function of the transverse radius (left) and the photon energy (right).

Only events with a polar angle $\left|\cos \theta_{K_{L}}\right|<0.77$ are retained, to minimize the photon absorption on the quadrupoles.

The proper time, $t^{*}$, distribution obtained from the selected sample is shown in fig. 7 (left) together with the fit result (the red thick solid line) in the range $8<t^{*}<26 \mathrm{~ns}$ using the function:

$$
f\left(t^{*}\right)=N_{0}\left(3 \pi^{0}\right) e^{-t^{*} / \tau} \epsilon_{\mathrm{acc}}\left(t^{*}\right)+f_{\mathrm{bck}} B\left(t^{*}\right)
$$


where $\epsilon_{\mathrm{acc}}$ is the signal acceptance, $B\left(t^{*}\right)$ is the bin content of the background shape from Monte Carlo, and $f_{\text {bck }}$ is a background scaling factor which is also a parameter of the fit (only background shape is evaluated from Monte Carlo). The blue line represents the $\sim 1.81 \%$ residual background shape: the two peaks before $8 \mathrm{~ns}$ are due to regeneration events and nuclear interactions on the beam pipe and on the internal wall of the DC, while after $25 \mathrm{~ns}$ the background increases since we are approaching the fiducial volume limit, near the calorimeter.

The fit result obtained with $\sim 46$ million events is $\tau=(50.56 \pm 0.14) \mathrm{ns}$ with a $\chi^{2}$-value of 50 for 54 degrees of freedom, fig. 7 (right) shows the fit residuals.

$\mathbf{x} 10^{2}$
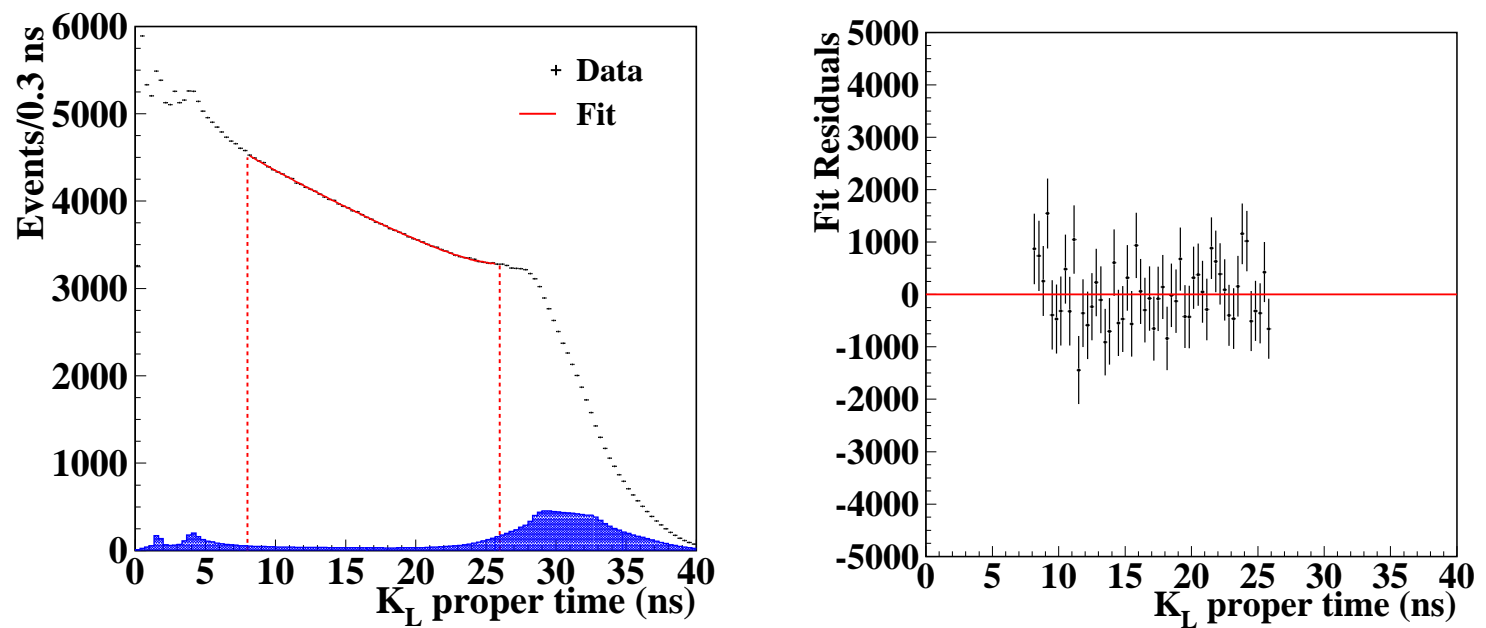

Figure 7: Left: Fit to the $K_{L}$ proper time distribution in the range 8-26 ns, the fit is shown as the thick solid red line, and the background shape from MC is the blue line; Right: Fit residuals.

Many effects distort the proper time distribution and have been corrected for. The uncertainty in the corrections is included in the systematic error on the $K_{L}$ lifetime, the main sources are shown in Table 2. The tagging efficiency as a function of the $K_{L}$ proper time is $\sim 70 \%$ and has a small linear

\begin{tabular}{|c|c|}
\hline Source & Systematic uncertainty \\
\hline Tagging efficiency & $0.17 \mathrm{~ns}$ \\
Preselection efficiency & $0.08 \mathrm{~ns}$ \\
Selection efficiency & negligible \\
Time Scale & $0.06 \mathrm{~ns}$ \\
Nuclear Interactions & $0.08 \mathrm{~ns}$ \\
\hline Total & $0.21 \mathrm{~ns}$ \\
\hline
\end{tabular}

Table 2: Summary of $K_{L}$ lifetime systematic uncertainties.

dependence on $l_{K}$ for $K_{L} \rightarrow 3 \pi^{0}$ decays. The correction for the $K_{L}$ lifetime is evaluated fitting this efficiency (fig. 7 , left). The preselection efficiency refers to the acceptance cuts: the calorimeter energy threshold of $20 \mathrm{MeV}$, and no cluster association to any tracks. The acceptance refers to the 
background rejection cuts and the time scale of the calorimeter calibration. The systematics on nuclear interactions includes uncertainties on the MC shape and on the normalization from the fit to data.

Finally, the preliminary result is:

$$
\tau_{K_{L}}=50.56 \pm 0.14_{\text {stat }} \pm 0.21_{\text {syst }} \mathrm{ns}=50.56 \pm 0.25 \mathrm{~ns},
$$

compatible with previous KLOE [12] measurements, still to be much improved.

The statistical error can be improved by decreasing the lower limit of the fit region, properly accounting for the $K_{L}$ beam losses on the regenerating surfaces. Moreover, adding the 2004 KLOE data set, the statistical error on the $K_{L}$ lifetime is expected to further decrease to $\sim 0.1 \mathrm{~ns}$. The systematic error arising from the tagging efficiency, due only to detector acceptance, is expected to decrease also.

\section{Absolute $K^{+} \rightarrow \pi^{+} \pi^{-} \pi^{+}$branching ratio studies.}

The measurement of $\mathrm{BR}\left(K^{+} \rightarrow \pi^{+} \pi^{-} \pi^{+}\right)$completes the KLOE program of precise and fully inclusive measurements of kaon dominant BR's. The most recent result, $\operatorname{BR}\left(K^{ \pm} \rightarrow \pi^{ \pm} \pi^{+} \pi^{-}\right)=$ $(5.56 \pm 0.20) \%$ [14] with a relative error $\sim 3.6 \%$, dates back to more than 30 years ago.

Two independent samples are used, tagging the $K^{+}$production by detection of the two-body decays $K \rightarrow \mu \nu$ and $K \rightarrow \pi \pi^{0}$ ( $\sim 85 \%$ of charged kaon decays) of negative kaons. The tagging procedure is as follows. We require a negative track (tagging kaon candidate) whose point of closest approach to the IP is inside a cylinder of with $10 \mathrm{~cm}$ radius and with $|z|<20 \mathrm{~cm}$. The kaon candidate decay point (a kink) must then satisfy $40<R_{\perp}<150 \mathrm{~cm}$, where $R_{\perp}$ is the transverse radial distance from the beam axis. The kaon momentum must be in the range $70<p(K)<130$ $\mathrm{MeV} / \mathrm{c}$.

Once the kaon candidate has been identified, we also require. 1). The candidate secondary track must have the same charge as the kaon. 2). The momentum difference between kaon and the secondary must satisfy $-320<\Delta p=\left|\mathbf{p}_{K}\right|-\left|\mathbf{p}_{\text {sec }}\right|<-120 \mathrm{MeV} / \mathrm{c}$. 3). The secondary momentum in the kaon rest frame, $p^{*}$, in the charged pion mass hypothesis, must be in the range:

$$
\begin{aligned}
225 & <p^{*}<245 \mathrm{MeV} / \mathrm{c} \quad \text { to select } K \rightarrow \mu v ; \\
195.5 & <p^{*}<213.5 \mathrm{MeV} / \mathrm{c} \quad \text { to select } K \rightarrow \pi \pi^{0} .
\end{aligned}
$$

as shown in fig. 8, left. The tagging efficiency is $\epsilon_{\text {tag }} \sim 25 \%$. From the measured properties of the tagging kaon, the positive kaon momentum, is obtained according to $\mathbf{p}_{K^{+}}=\mathbf{p}_{\phi}-\mathbf{p}_{K^{-}}$.

The decay products of the kaons have a very low momentum, less than $200 \mathrm{MeV} / \mathrm{c}$, and curl up in the KLOE magnetic field $(0.52 \mathrm{~T})$. This results in the reconstruction of many broken tracks and fake vertices. If the kaon is requested to decay before reaching the inner wall of the DC, the kaon track is not detected and the quality of the reconstruction improves if no more than three reconstructed tracks are requested. At least two tracks reconstructed in the DC (pion candidates) are required, then if their backward extrapolations make a vertex along the kaon path before the inner wall of the DC (the geometrical acceptance is $\sim 26 \%$ ), the missing mass of the decay in the pion mass hypothesis is evaluated. The fig. B shows the comparison between data and Monte Carlo of the squared missing mass spectrum and the MC background contribution is superimposed. 

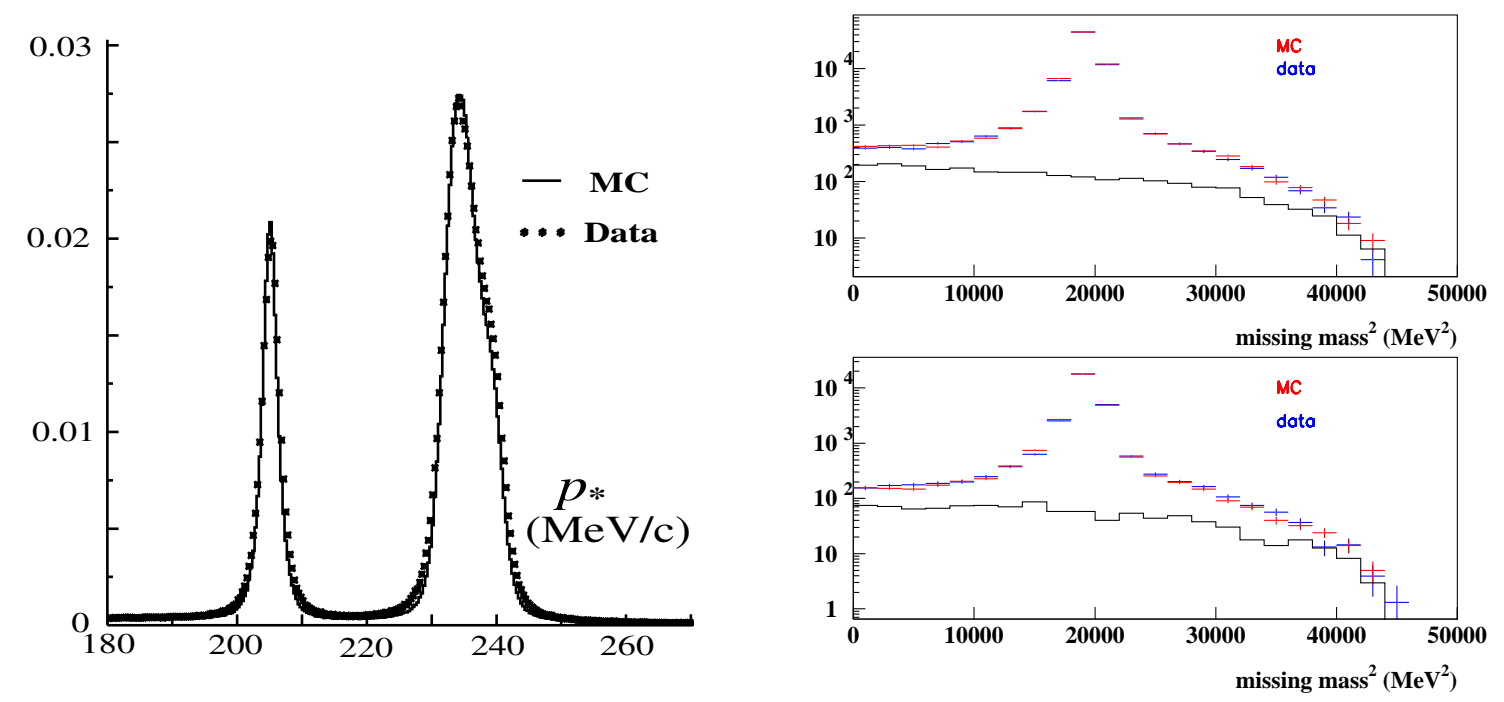

Figure 8: Left: Momentum spectrum in the kaon rest frame of the negative charged decay particle, assuming the particle has the pion mass for data (dots) and MC (lines). The distribution is normalized to unity. The two peaks correspond to pions and muons from $K^{-} \rightarrow \pi^{-} \pi^{0}(205 \mathrm{MeV} / \mathrm{c})$ and $K^{-} \rightarrow \mu^{-} \nu_{\mu}(236 \mathrm{MeV} / \mathrm{c})$. The muon peak is broadened by the use of the incorrect mass. Right: Squared missing mass distribution for $\mu v$ tag (top) and $\pi \pi^{0}$ tag (bottom). In black the background, from MC.

The acceptance will be evaluated from Monte Carlo, and corrections will be applied to take into account data-MC differences in tracking. The work is in progress. KLOE can reach a statistical relative error of a few $\times 0.1 \%$.

\section{References}

[1] M. Adinolfi et al. (KLOE Collaboration), Nucl. Instrum. Methods A488 (2002) 51.

[2] M. Adinolfi et al. (KLOE Collaboration), Nucl. Instrum. Methods $\mathbf{A 4 8 2}$ (2002) 364.

[3] M. Adinolfi et al. (KLOE Collaboration), Nucl. Instrum. Methods $\mathbf{A 4 9 2}$ (2002) 134.

[4] F. Ambrosino et al. (KLOE Collaboration), Nucl. Instrum. Methods A534 (2004) 403.

[5] F. Ambrosino et al. (KLOE Collaboration), J. High Energy Phys. 04 (2008) 059.

[6] F. Ambrosino et al. (KLOE Collaboration), Phys. Lett. B 636 (2006) 173.

[7] S. Aoki et al., Astron. Astrophys. 105 (1982) 359.

[8] A. Alavi-Harati et al. (FNAL KTeV Collaboration), Phys. Rev. D 67 (2003) 012005.

[9] Ed. Blucher (FNAL KTeV Collaboration), Talk at KAON09 Conference, Tsukuba, Japan.

[10] A. Lai et al. (CERN NA48 Collaboration), Phys. Lett. B 537 (2002) 28.

[11] F. Bossi et al. (KLOE Collaboration), Riv. Nuovo Cim. 031 (2008) 531.

[12] F. Ambrosino et al. (KLOE Collaboration), Phys. Lett. B 626 (2005) 15.

[13] F. Ambrosino et al. (KLOE Collaboration), Phys. Lett. B 632 (2006) 43.

[14] I.H. Chiang et al., Phys. Rev. D 6 (1972) 1254. 